\title{
" Puede respirar, sniff ? " Les soignants face aux défis des interactions avec des publics migrants
}

\section{"Puede respirar, sniff?" Caregivers facing the challenges of interactions with migrant audiences}

\author{
Anna-Claudia Ticca ${ }^{1}$ \\ ${ }^{1}$ Laboratoire ICAR UMR 5191 (CNRS, ENS de Lyon, Université Lumière Lyon 2), France
}

\begin{abstract}
Résumé. Cette contribution vise à mettre en lumière les compétences interactionnelles et relationnelles des professionnels de soins dans la prise en charge de patients allophones. Un aspect important de ces interactions observées est le travail d'élaboration de l'intercompréhension, caractérisé par des séquences de reformulation, répétition, réparation, etc., en français et dans d'autres langues, avec le recours à des pratiques multimodales (i.e. verbales et non verbales). À travers l'analyse de segments d'interactions filmées en situation, cette étude met en lumière les savoir-faire relationnels et interactionnels des soignants, qui jonglent avec l'utilisation d'une ou plusieurs lingua franca et d'autres ressources interactionnelles afin de gérer la consultation dans ses dimensions informationnelle, relationnelle et de soin. Renouveler son répertoire de ressources représente un défi nécessaire pour les professionnels du soin qui interagissent avec des publics migrants.
\end{abstract}

\begin{abstract}
This paper aims at highlighting caregivers' interactional and relational skills when they interact with allophone patients. In such cases, an important work to build inter-comprehension is made through, for instance, sequences of reformulation, repetition, repair, etc., both in French and in other languages and by restoring to multimodal practices (i.e. verbal and non-verbal). Through the analysis of extracts of video-recorded interactions, this study sheds light on the relational and interactional savoir-faire of caregivers, who juggle one or more lingua franca and other interactional resources in order to manage the consultation in its informational, relational and care dimensions. Renewing one's repertoire of resources represents a necessary challenge for healthcare professionals who interact with migrant audiences.
\end{abstract}




\section{Introduction}

Les recherches en interaction basées sur l'observation de données empiriques audiovisuelles représentent une ressource importante pour appréhender les activités sociales dans leur dimension langagière multimodale (c'est-à-dire, verbale et non verbale). Elles permettent de saisir le caractère incarné et multidimensionnel de l'action sociale et d'en observer les microdétails de l'activité communicative dans leur matérialité interactionnelle. Au cours des dernières années, un grand nombre de recherches dans ce champ a œuvré en faveur d'une diffusion des résultats avec une claire orientation pédagogique. Ceci se traduit par une valorisation, sous différents formes et modalités, des analyses scientifiques pour des publics non exclusivement académiques.

La recherche REMILAS ${ }^{1}$ (Refugiés, Migrants et leurs LAngues face aux services de Santé) en est un exemple, initialement pensée pour rendre compte du fonctionnement des interactions plurilingues avec des publics migrants et allophones dans le domaine de la santé. Au cours de ce projet, il est devenu évident que la publication des résultats dans des revues scientifiques en linguistique, parfois de niche, laissait inévitablement à l'écart les professionnels concernés (soignants, interprètes, travailleurs sociaux, etc.) et susceptibles d'être intéressés par les études menées.

Cet article s'inscrit dans cette ligne de réflexion et oriente les analyses proposées dans une visée formative, à destination des professionnels du soin notamment. Les interactions spontanées enregistrées ${ }^{2}$ dans le cadre de la recherche REMILAS offrent l'opportunité d'observer la pratique professionnelle telle qu'elle se réalise en situation, dans un environnement spécifique à chaque interaction, et dans lequel la signification se co-construit localement, dans l'ici et maintenant de l'action communicative.

À partir d'extraits d'interactions filmées dans un dispensaire public et dans une Permanence d'Accès aux Soins et à la Santé (PASS) en France, nous analyserons des séquences d'interactions où médecins et patients, qui ne partagent pas la même langue maternelle, construisent l'intercompréhension en mobilisant des ressources sémiotiques multiples et diverses. Ces observations mettront en lumière d'une part les difficultés rencontrées par les participants lors de ces rencontres qui se déroulent sans l'accompagnement de tiers traducteurs, d'autre part les compétences et savoir-faire des soignants mises en œuvre dans la prise en charge des patients allophones. Certaines expressions attestées dans les enregistrements telles que « have you dolor ?» ou « puede respirar sniff», où la soignante recourt à son répertoire linguistique afin de communiquer avec un patient non francophone, synthétisent les difficultés des professionnels de la santé dans leur pratique quotidienne mais aussi leur capacité à mettre en place des modalités communicatives alternatives. Comprendre la complexité de l'agir contribue à construire des connaissances sur la pratique professionnelle, et, plus spécifiquement, sur les compétences interactionnelles et relationnelles, qui sont ensuite réinvesties en formation.

L'article présentera d'abord la perspective théorique concernant l'analyse des interactions et ses enjeux pour la formation (2); il exposera ensuite les interactions en santé dans un contexte plurilingue (3) ainsi que les spécificités des consultations en santé générale (4). La section analytique (5) sera suivie par une réflexion sur les implications des analyses pour la formation (6).

\footnotetext{
${ }^{1}$ http://icar.cnrs.fr/projet-remilas/

${ }^{2}$ Le corpus des vidéo-enregistrements se compose de 91 interactions.
} 


\section{La recherche en interaction et son apport pour la formation}

L'analyse des interactions s'opère par une méthode d'observation granulaire des comportements multimodaux des participants, où paroles, gestes, regards, mouvements du corps, manipulations d'objets, etc., sont analysés dans leur déploiement synchrone et coordonné. De même, le regard analytique se pose sur la co-production des actions interactionnelles et observe le processus interprétatif que chaque participant opère sur l'activité en cours. En effet, à travers leurs actions les participants manifestent leur compréhension de l'événement dans lequel ils sont engagés et, avec leurs actions successives ils contribuent à le faire avancer (Mondada, 2006, Traverso 2016). Dans cette perspective, la pratique professionnelle est observée en tant que processus interactionnel, intégrant plusieurs éléments tels que l'action des partenaires ainsi que le contexte écologique situé. Celui-ci inclut l'espace de l'interaction, la configuration des personnes et des objets dans cet espace, les ressources sémiotiques mobilisées (y compris la ou les langues) lors des échanges (Duranti \& Goodwin, 1992, Mondada, 2006). À travers l'examen des séquences d'interactions, l'attention se concentre sur des moments récurrents dans les échanges et qui semblent favorables à l'identification des compétences ou des savoir-faire professionnels, favorisant par conséquence la compréhension de l'activité professionnelle (Vinatier 2013 ; Pekarek et al 2017). C'est le cas des situations où la conversation se complique et les participants s'engagent dans des séquences de réparation ou correction, reformulent leurs tours de parole ou ceux des co-participants, et mobilisent des ressources diverses pour résoudre l'impasse et permettre la progression de l'interaction afin d'atteindre les objectifs de la rencontre (Traverso, 2017). Dans ce sens, nous sensibilisons les professionnels à la place du langage au travail (cf. Filliettaz 2014).

D'autres études sur la communication plurilingue, cette fois-ci en présence d'interprètes, ont montré que ces professionnels peuvent s'engager dans des échanges dyadiques, avec seulement un des participants à l'interaction. Or, bien que ces interventions spontanées soient a priori non recommandées si l'on regarde la charte de l'interprétariat ${ }^{3}$, elles se révèlent souvent efficaces pour résoudre ou anticiper des problèmes de communication, les incompréhensions ou les malentendus (Niemants et al, 2015 ; Ticca \& Traverso, 2015a). Apprendre à accepter la «transgression » d'une norme afin de contribuer à la réussite d'un échange conversationnel peut rassurer les interprètes, dont la pratique se trouve souvent en tension entre déontologie et adaptation.

Dans le cadre de nos recherches, cette perspective analytique micro est accompagnée par une ouverture du regard sur le contexte plus large des interactions, développé à travers un travail ethnographique qui inclut des échanges prolongés avec les professionnels de soin et les interprètes. Appréhender les enjeux et les attentes des participants vis-à-vis de la rencontre sociale nous permet, en effet, de mieux saisir les significations produites lors des interactions et de pouvoir ensuite aider les professionnels à adapter leur pratique et à modifier leur propre manière d'inter-agir.

\section{L'interaction plurilingue en santé}

Le déplacement des personnes à travers les frontières, souvent à cause de persécutions subies dans leurs pays d'origine, contribue à rendre nos sociétés de plus en plus multilingues et multiculturelles. Dans le domaine de la santé, cela se traduit par la rencontre entre cultures médicales distinctes, à lesquelles s'ajoutent les différences dans les usages linguistiques qui

\footnotetext{
3 Charte de l'interprétariat médical et social professionnel en France https://www.unaf.fr/IMG/pdf/charte-signee-scan19-12-2012.pdf
} 
émergent lors des consultations. Il existe une riche littérature soulignant les retombées de ces diversités sur la pratique clinique et sur la relation entre médecin et patient, qu'il soit allophone ou alors locuteur de la même langue du médecin mais provenant d'un autre pays (Singy et al, 2010). Résoudre graduellement les « énigmes » qui se produisent en interaction représente un défi pour les professionnels, qui doivent souvent s'adapter aux styles communicatifs et aux postures culturelles de leurs patients (Roberts, 2010). Selon l'autrice, il ne s'agit pas de casser des barrières, puisque ceci évoque une action forcée et unidirectionnelle produite par la culture dominante envers celle minoritaire du patient étranger. Dans cette perspective, les langues sont considérées comme des possibilités plus que des obstacles à l'échange. Cependant, la réalité de la pratique clinique, avec ses contraintes temporelles et ses ressources matérielles et humaines limitées, oblige à dessiner un panorama dont la réalité est bien moins idéalisée que celle présentée ici. Qu'il s'agisse de médecine somatique ou de santé mentale, la gestion du plurilinguisme dans le soin est complexe et implique plusieurs dimensions du phénomène. Si la présence de tiers médiateurs est souvent considérée comme la solution pour faire face aux diversités linguistiques et culturelles (sur les résistances et ambivalences des soignants quant au recours à l'interprète professionnel dans les consultations, voir Bischoff et al. 2003 ; Weber \& Molina, 2003 ; Goguikian Ratcliff, 2010), leur présence n'est pas toujours assurée. Le personnel de santé est souvent obligé de pratiquer sans interprète, en mobilisant une lingua franca ou d'autres moyens pour communiquer (comme des outils numériques tels que Google traduction, Traducmed, ou des applications de traduction sur smartphone), ou alors de compter sur la traduction non spécialisée des personnes qui accompagnent les patients au cabinet. Dans le cadre de la recherche REMILAS, nous avons attesté plusieurs modalités mises en œuvre par les soignants dans la prise en charge de patients allophones (consultations sans interprète, avec interprète professionnel, en présence ou par téléphone, avec interprète improvisé, avec l'utilisation d'outils de traduction numériques, avec une lingua franca, etc.). Dans certains cas, ces modalités se combinent, impliquant la mobilisation de plusieurs ressources selon le besoin du moment (voir entre autres Ticca \& Traverso, 2017 ; Piccoli et al., 2019). Parmi les raisons qui conduisent à interagir sans interprète, il faut mentionner la pénurie de ressources financières destinées au volet «interprétariat» (c'est en effet une prérogative des établissements de fournir le service d'interprétariat lors des consultations). De plus, dans le cas des consultations en santé somatique notamment, il y a également un problème de type organisationnel. De fait, les médecins ne connaissent pas toujours à l'avance l'identité de leurs patients et se retrouvent face à la diversité linguistique sans possibilité d'anticiper adéquatement leur accueil.

\section{La consultation en médecine générale}

La visite médicale a fait l'objet de nombreuses études qui décrivent le déroulement et l'organisation de ce type d'interaction institutionnelle (voir Ticca \& Traverso, 2015b pour une revue des études dans le domaine). Dans ce cadre, les asymétries épistémiques sont à la base de la rencontre sociale, et les rôles et les activités des interlocuteurs déterminés par le contexte (Drew \& Heritage, 1998, Lacoste, 1993). Organisée en phases (ouverture, présentation du problème, interrogatoire, auscultation, diagnostic, prescription, etc., Cosnier et al. 1993), les activités qui ont lieu dans chacun de ces moments sont toutefois négociées et co-construites et les participants ont une marge de manœuvre leur permettant de s'adapter aux co-participants et aux circonstances locales du déroulement de l'interaction (Lacoste 1980 ; Heritage \& Maynard, 2006).

A ces spécificités nous en ajoutons d'autres, concernant les finalités et les enjeux des consultations. En effet, une visite généraliste qui vise à réaliser un bilan de santé, propédeutique à l'inscription dans le système national de santé d'un primo-arrivant n'ayant 
pas des droits ouverts, sera organisée et menée différemment d'une visite dont l'objectif est celui d'établir un diagnostic et donner un traitement à une personne présentant des symptômes et nécessitant un soin ou un avis médical. Les questions posées ainsi que les réponses attendues seront donc adaptées au contexte et aux buts de la consultation.

Les analyses suivantes, basées sur l'observation détaillée des activités multimodales des participants, tendront aussi compte d'éléments contextuels tels qu'ils se manifestent dans la matérialité des interactions.

\section{Les données et le contexte}

Les consultations sélectionnées pour cette étude ont été enregistrées dans deux situations distinctes : des consultations dans une PASS et dans un dispensaire public en France.

Les consultations à la $\mathrm{PASS}^{4}$ visent à produire un premier bilan de santé des personnes primoarrivantes en France, premier pas pour accéder à l'ouverture de droits à la santé. En cas de problèmes de santé, des soins sont également prévus et dispensés lors de la même visite. Ces entretiens servent également à établir la pertinence d'un accompagnement social et ou psychologique / psychiatrique pour le patient, qui sera activé par le même médecin.

Quant au dispensaire ${ }^{5}$, les consultations sont dédiées au soin des patients. Cependant, l'interaction peut se nourrir d'échanges non strictement médicaux. Puisqu'ils ne sont pas fonctionnels aux objectifs de la visite, nous verrons que les patients peuvent manifester une certaine résistance au développement des contenus de ces échanges.

\subsection{Plusieurs langues, plusieurs ressources}

Les analyses suivantes se focalisent sur les procédés multimodaux mis en œuvre par les participants au fil de leurs échanges pour construire l'intercompréhension. Elles mettront ainsi en lumière la dimension relationnelle de ces consultations telle qu'elle émerge en situation. Les extraits vidéo utilisés pour les analyses ont été transcrits en suivant les conventions explicitées en annexe.

\subsubsection{La réparation multimodale}

Le cas suivant, issu d'une consultation entre le jeune patient Antonon (ANT), sa mère Vera (VER) et la médecin (DOC), se déroule en ayant recours à plusieurs langues (français, italien, espagnol) ainsi qu'à la mobilisation de gestes et d'autres ressources linguistiques, comme les onomatopées. Dans la transcription, une traduction en français est insérée en italique après les segments originaux en langue étrangère, signalés en rouge dans le texte.

Antonon est né en Italie et l'italien est sa langue de socialisation, utilisée pour communiquer avec sa mère, d'origine macédonienne. Il manifeste également des compétences en français,

${ }^{4}$ Il s'agit de 13 consultations avec 2 médecins, dont 6 réalisées en anglais lingua franca pour l'ensemble des participants. Dans 3 cas (avec 2 patients albanophones et 1 Macédonien), une personne accompagnante a pris en charge la traduction en anglais. (Le reste des consultations se sont déroulées en français).

${ }^{5} \mathrm{Ce}$ corpus consiste en 10 consultations avec 2 médecins, dont 5 réalisées en anglais lingua franca pour l'ensemble des participants et 1 en espagnol langue étrangère pour le médecin. Dans 4 cas (avec 1 patient arabophone et 3 albanophones), une personne accompagnante a pris en charge la traduction en français. 
ainsi que Vera. Quant à la soignante, elle recourt parfois à l'espagnol pour interagir avec le garçon.

La médecin ouvre l'interrogatoire en s'adressant à Antonon, assis devant elle avec sa mère.

\section{1. «Atchou »}

((lignes 01-09: salutations))
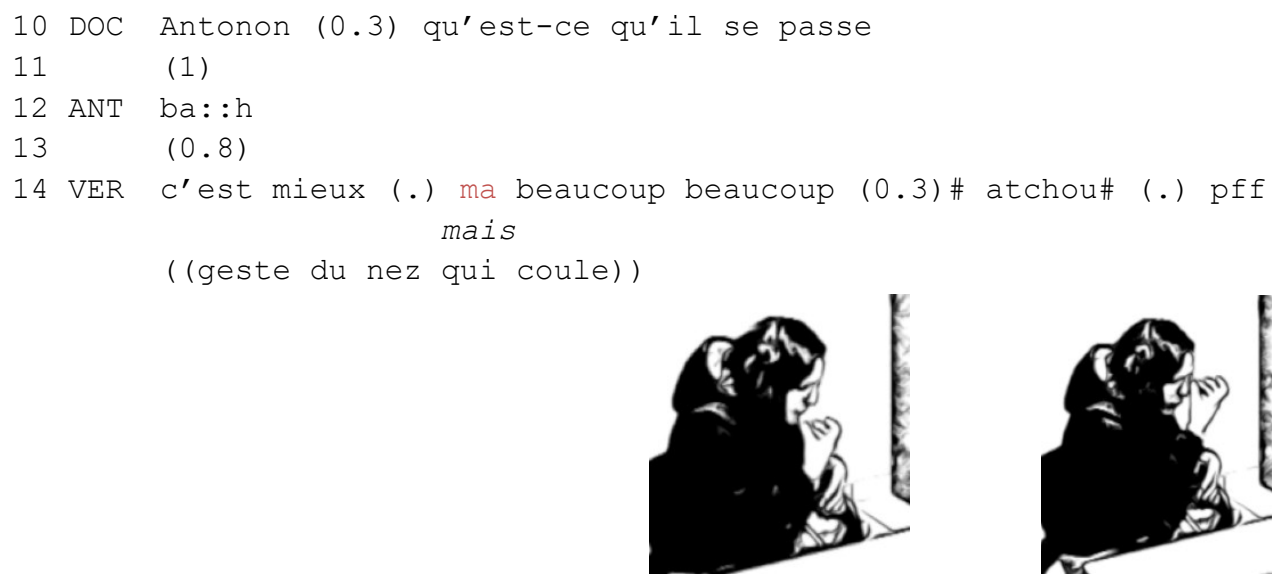

\#1


17

$(0.8)$

18 VER la nuit: : la journée: la- toute la journée et toute la nuit: 19 $(0.5)$

Vera produit une onomatopée afin de décrire le symptôme du garçon (atchou, 1. 14), l'accompagnant avec des gestes que l'on peut définir d'iconiques (Im. 1-2). La médecin accepte ce procédé : non seulement elle ne proposera pas le terme en français mais elle recyclera la même pratique multimodale dans la suite, pour demander, de manière syntaxiquement succincte, si la nuit aussi le « nez coule» (1. 16, Im. 3-4). La réponse arrive après une brève pause, permettant la poursuite de l'interrogatoire.

Contrairement à ce que nous venons d'observer, quelques instants plus tard le médecin corrigera une production verbale concernant la durée des symptômes. Il s'agit plus précisément d'une réparation du tour antérieur réalisée à travers une traduction en français du mot en italien tre - trois. Fréquent dans les interactions plurilingues, ce procédé est utilisé pour montrer sa propre compréhension ainsi que pour s'assurer de la compréhension mutuelle (Svennevig, 2004, Piccoli, 2020). 


\section{2. «Atchou »}

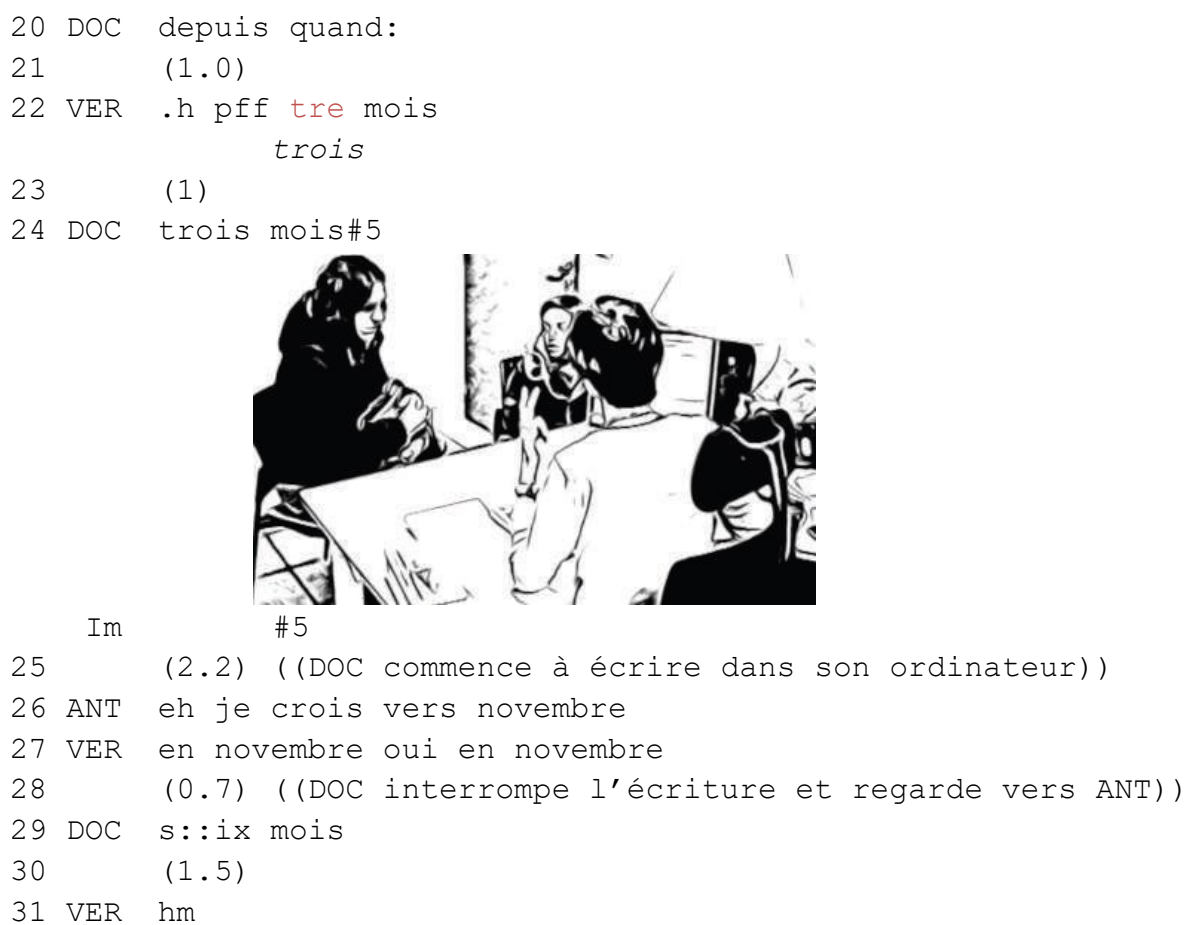

Ici, la réparation est accompagnée par le geste de la main indiquant le chiffre 3 (image 5). Cette réparation multimodale permet aux interlocuteurs de visualiser la compréhension du médecin et éventuellement de la corriger à leur tour. C'est en effet ce qui se passe dans la suite. Antonon reprend la parole après quelques instants de pause (1. 25), et corrige l'indication temporelle co-élaborée par sa mère et la soignante, qui, vraisemblablement, est en train de l'écrire dans l'ordinateur (1. 25). Finalement, la durée des symptômes d'Antonon sur laquelle l'ensemble des participants est d'accord est de six mois (1. 27-31).

Quelques instants plus tard, la médecin reviendra sur les symptômes et utilisera encore une fois «atchou » pour indiquer le problème de l'enfant. Ce n'est que lors d'une troisième référence à ce même problème (extrait non montré ici) que la soignante aura recours au terme « éternuer» en l'associant à l'onomatopée.

La réparation des segments de parole des co-participants ne s'opère donc pas systématiquement, mais elle apparaît de manière ciblée. Dans le cas présenté ici, elle a été effectuée afin d'éviter de potentiels malentendus sur des informations médicalement importantes, comme la date de début des symptômes.

\subsubsection{Le bricolage linguistique}

La résolution des problèmes d'intercompréhension passe également par l'utilisation d'une troisième langue. L'extrait suivant, tiré de la même consultation, en est un exemple. Les participants sont engagés dans la description des symptômes d'Antonon ${ }^{6}$.

\footnotetext{
${ }^{6}$ Dans cet extrait, nous avons recommencé la numérotation des lignes à partir de zéro (00) après la
} ligne 99. 


\section{3. « Puede respirar?»}

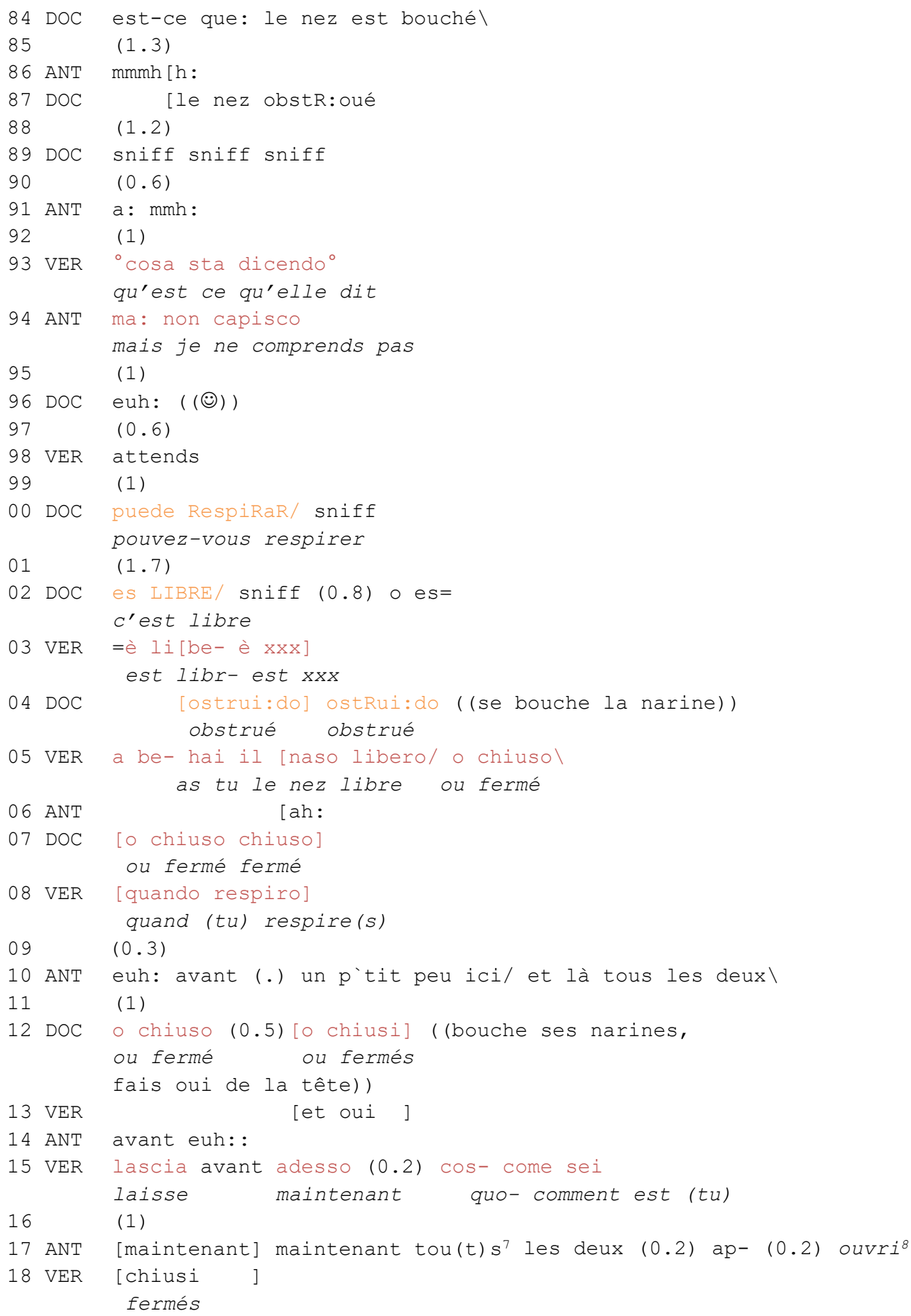

${ }^{7}$ Antonon prononce ici la $t$.

${ }^{8}$ Ici nous semble qu'Antonon construit l'adjectif « ouverts » en s'inspirant de l'italien aperti. 
Dans cette séquence différents procédés sont mis en œuvre par la soignante afin de se faire comprendre. Elle pose une première question en français (1. 84) mais les interlocuteurs ne comprennent pas (une longue pause puis une hésitation de la part de Antonon suivent la question). Un deuxième essai est alors proposé, cette fois-ci avec un énoncé présentant une simplification syntaxique, un changement lexical et une adaptation prosodique concernant la modulation de la voix (« le nez obstR:oué », 1. 87). Encore une fois, les co-participants ne répondent pas, ce qui conduit la soignante à une ultérieure tentative, où elle incarne le symptôme en simulant l'effet respiratoire du problème (sniff sniff sniff, 1. 89). Toutefois, ces efforts n'aboutissent pas au résultat attendu (voir les pauses puis l'échange en italien entre mère et enfant, 1. 90-95) et après un petit sourire (peut-être de découragement) la soignante mobilise ses connaissances en langue espagnole ("puede respirar/ sniff», 1. 00). La production de ce tour de parole est ralentie, avec un volume augmenté, des traits typiques du foreign talk. La suite, toujours en espagnol, après une autre réponse manquée (1. 1), présente les même caractéristiques (es LIBRE /sniff). À ce moment-là, Vera semble comprendre : elle traduit en italien ce qui vient d'être dit $(1.3,5,8)$ et Antonon répond enfin au médecin, en français (1. 10). A noter également la répétition par la médecin du mot italien chiuso (tr. fermé, ou obstruido en espagnol) utilisé par Vera dans sa traduction, comme à vouloir indiquer que c'était celui-ci le mot qu'elle recherchait ou aurait utilisé elle-même, et avec lequel, en dernière instance, elle valide le choix de Vera et, par conséquence, sa reformulation en italien ${ }^{9}$.

L'utilisation d'onomatopées et des gestes pour construire la signification a été attestée tout au long de cet entretien, ainsi que d'autres présentes dans le corpus. Si l'efficacité de ces procédés n'est pas mise en question, ainsi comme la possibilité qu'elles offrent aux participants d'établir une relation de proximité (voir paragraphe ci-dessous), ce sont leur emploi et leur adaptation continue aux circonstances du moment qui ont représenté un défi pour les participants. De fait, des manifestations de découragement et agacement de la part de la soignante ont surgi vers la fin de l'entretien suite à l'impossibilité de se faire comprendre. C'était grâce à l'utilisation de l'Internet que l'impasse a pu être surmontée. Interrogée sur la gestion de l'interaction interlinguistique, la soignante déplore l'absence d'un accompagnement spécialisé pour pallier ces difficultés.

\subsubsection{La dimension relationnelle}

L'extrait suivant concerne une interaction en anglais à la PASS avec un jeune demandeur d'asile nigérien récemment arrivé en France. Il a été envoyé chez le médecin par l'assistante sociale de la même structure à cause de ses problèmes de santé (grippe et mal-être généralisé). Dans cet entretien, où l'état psychophysique du patient est assez fragile, ce qui retient notre attention est la gestion par la soignante des moments dans lesquels les frontières entre santé somatique, santé mentale et vulnérabilité sont assez floues.

Lors de l'interrogatoire, juste après avoir recueilli l'information relative aux soucis de santé saisonniers, la médecin questionne le patient sur la présence d'autres problèmes physiques ${ }^{10}$ :

\footnotetext{
${ }^{9}$ Faute d'espace, nous ne pourrons pas développer ici la question liée aux interventions de Vera, qui corrige et réoriente les réponses de l'enfant-patient, complexifiant la gestion de l'interrogatoire et des informations y produites.

${ }^{10}$ Les images 3 ci-dessous sont tirées des vidéos capturées par deux caméras distinctes dans un même moment, ce qui offre deux vues de la même situation.
} 


\section{4. « Complaint»}

01 DOC do you have any: : other complaint/ vous avez d'autres plaintes

$02 \quad(0.8)$

03 DOC other problem/ autre problème

$04 \#(2.3)$ ((se touche le corps à la hauteur de son épaule))

Im

05 SAM

06

07 SAM \# yeah $^{\circ}$

ouais

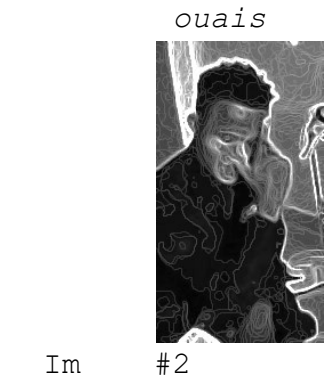

Im

08 DOC I ne- \#I didn't understand ((;))

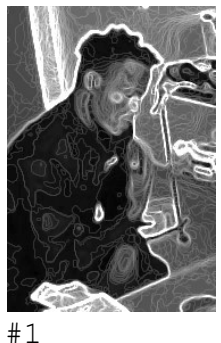

tch ${ }^{\circ}$ I guess I- is personal with me ${ }^{\circ}$ sniff

tch je pense je- c'est personnel avec moi sniff

(1.5) ((porte sa main sur le visage et ferme les yeux))

je ne- je n'ai pas compris

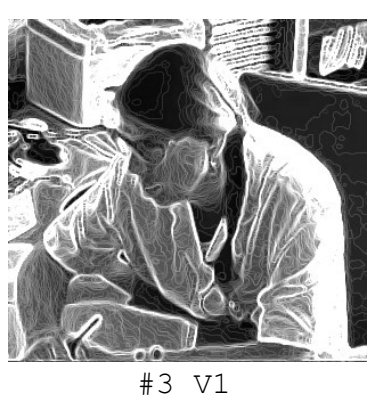

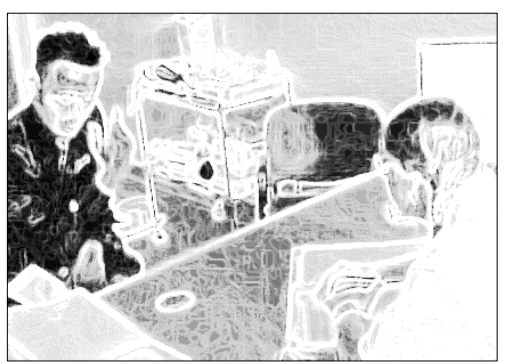

\#3 V2

09 SAM I said any other thing is- is within me it's personal so j'ai dit que tout autre chose est- est dedans moi c'est personnel donc

10 (0.3) I don't think I want to say (it with someone) (-d) xxx (0.3) je ne pense pas vouloir dire (ça avec quelqu'un) (d-) $x x x$

$11 \quad(1.3)$

12 DoC stay with someone again/

rester/être avec quelqu'un de nouveau 


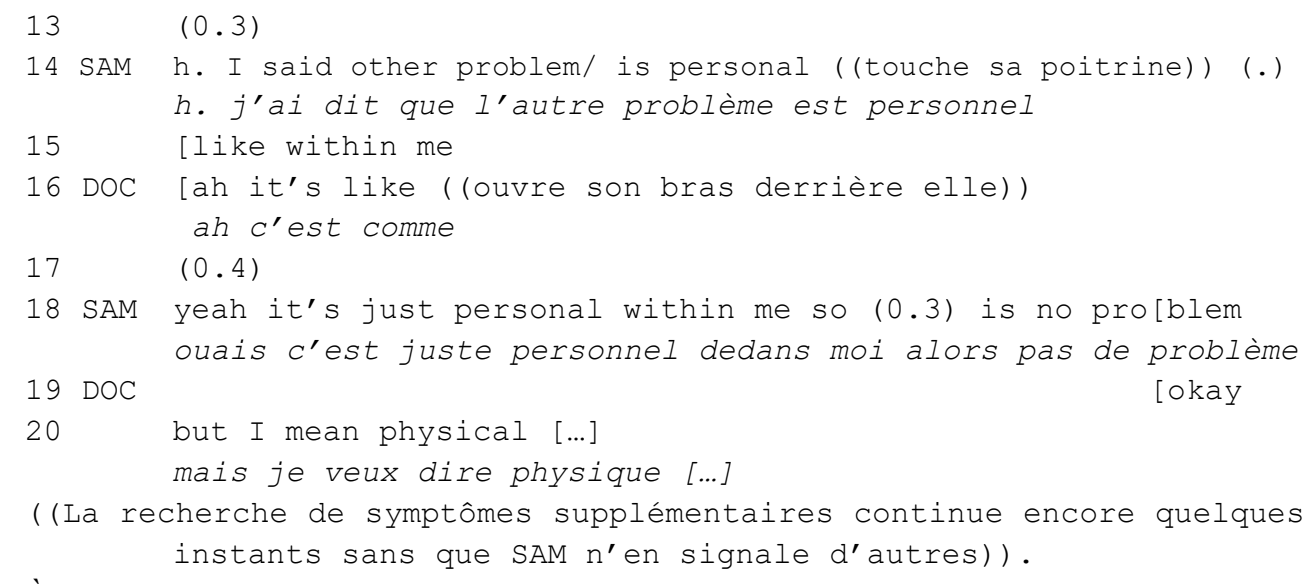

À la question sur la présence d'autres « plaintes » ou problèmes (1. 1), le patient commence à répondre de manière incarnée, en se touchant la poitrine (1. 4) et annonçant avoir des questions personnelles (1. 5). La souffrance de Sam est ici remarquable, manifestée pas sa gestualité aussi bien que sa locution (voir Im. 1-2). La médecin, qui le regarde attentivement, commence ensuite à parler mais elle se corrige en avouant n'avoir pas compris $(1.8, \operatorname{Im} .3)$. Dans les tours qui suivent, Sam précise avoir des soucis personnels dont il préfère ne pas parler (1. 9-10, 14-15, 18). Tout au long de cet échange, la soignante suit l'activité du patient même au moment où elle comprend que le mal être de Sam n'est pas strictement somatique (1. 12 et $\left.16^{11}\right)$. De fait, elle attend la fin de l'explication du patient pour préciser que le type de problème recherché est d'ordre physique (1.19).

Cette action de recadrage, que nous avons attestée aussi dans d'autres consultations enregistrées ${ }^{12}$, vise à réorienter les contenus produits par les patients afin de les rendre conformes aux questions posées ou aux attentes des soignants. Dans ce cas spécifique, un moment délicat de l'interrogatoire avec un patient visiblement vulnérable et en souffrance, la médecin manifeste un savoir-faire relationnel, en donnant la priorité à ce que l'interlocuteur a à dire avant de s'orienter vers la progression de l'interrogatoire somatique. Sa posture corporelle, orientée vers le patient, la modulation de la voix ainsi que le sourire, manifestent son empathie envers le patient. Ces instants d'attention relationnelle, fréquents dans l'interaction, pourraient être à l'origine du dévoilement des problèmes personnels de la part de Sam vers la fin de la consultation (données non montrées).

Comme nous l'avons souligné auparavant, le contexte de l'interaction joue un rôle dans l'interprétation et la construction des contenus interactionnels. Une question déplacée peut générer un malentendu ou alors, comme dans le cas suivant, un évitement ou une résistance à poursuivre l'activité proposée.

Juste avant la situation illustrée ci-dessous, la médecin a expliqué le traitement à suivre et a remis les ordonnances au patient. Ces activités, qui ont typiquement lieu dans les phases finales de la consultation, orientent les participants vers sa clôture. Dans l'extrait, alors que le patient est un train de lire un des documents remis et que la soignante en remplit un autre, celle-ci pose une question d'ordre non médical, qui se manifestera comme problématique.

${ }^{11}$ La particule « ah » en début de tour de parole montre son changement d'état de non-compréhension à compréhension (Heritage, 1984). Le mouvement du bras également semble indiquer un moment dans le passé, qu'elle semble avoir mis en relation avec les problèmes personnels mentionnés.

${ }^{12}$ Les nombreuses analyses collectives au sein de l'équipe REMILAS ont en effet permis de relever de manière récurrente le phénomène du recadrage dans les consultations médicales. 


\section{5. «Why do you leave Nigeria »}

01 DOC may I ask you \#why do you leave Nigeria\# peux je vous demander pourquoi vous avez quitté le Nigeria

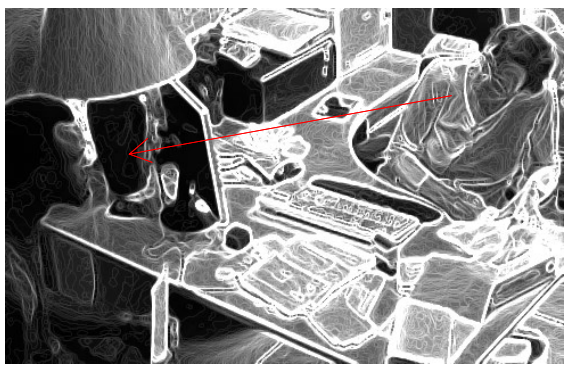

\#1

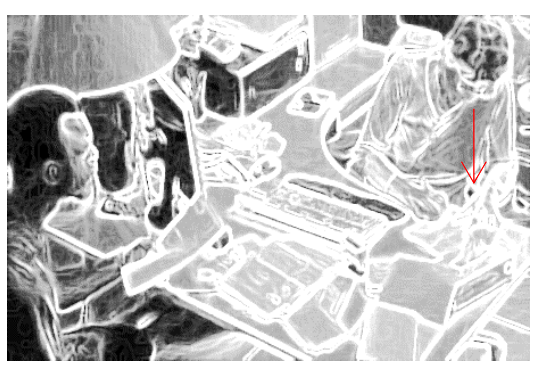

\#2

$02 \quad(0.3)$

$03 \mathrm{PAT} \mathrm{hm} /$

04 DOC may I ask you (.) why you have 1- you have left Nigeria peux je vous demander (.) pourquoi vous avez qu- vous avez quitté le Nigeria

05 PAT ah xxx is a very big long story I eh

06 DOC uh $\mathrm{hm} /=$

07 PAT =is a very big long story c'est une histoire très longue ((le téléphone fixe commence à sonner))

08 DOC yes I understand what you are saying (:) [but oui je comprends ce que vous dites mais

09 PAT

[you see/

vous voyez

\section{$10 \quad(0.3)$}

11 DOC what was $\mathrm{v}-$ the main reason

quelle était $\mathrm{v}$ - la raison principale ((en se tournant vers le téléphone))

12 (1.5)

13 PAT the main [reason

14 DOC [mmh\# attends oui allô ((au téléphone))

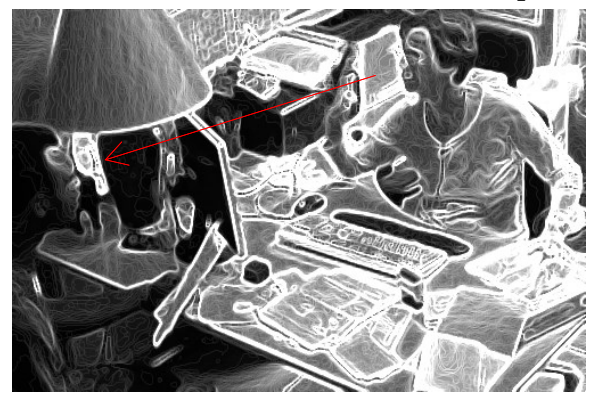

\#3

((conversation téléphonique de 20 sec.))

16 DOC oui what was the main reason ((en se retournant vers PAT)) quelle était la raison principale

((suit le récit de son histoire pendant $1 \mathrm{~min}$ et $30 \mathrm{se.}$ )) 
Deux aspects principaux qui découlent de cet extrait méritent d'être soulignés : d'abord, concernant les contenus verbaux de l'échange, on observe une première manifestation de résistance à la question initiale. En effet, celle-ci reçoit deux fois de suite la même réponse ( « is a very big long story »; " you see », $1.5,7,9$ ) qui manifeste, bien qu'implicitement, l'indisponibilité à une élaboration correspondante aux attentes émanant de la question. Ensuite, la relance de la soignante, visant à circonscrire le périmètre de la réponse en le limitant à la raison principale (1. 11), ne semble pas saisir et/ou prendre en compte l'indisponibilité du patient que nous venons de mentionner.

Ce qui attire notre attention est également la posture corporelle de la soignante, dont l'orientation attentionnelle est partagée entre le patient, l'écriture et le téléphone (Im. 1 - 3). Compte tenu du caractère sensible du sujet abordé, de la résistance manifeste du patient aussi bien que du moment (i.e. la conclusion de la visite) dans lequel ce sujet est traité, l'action de la soignante n'apparaît pas préparée efficacement. D'autant plus que l'évocation de situations potentiellement traumatiques, comme celles liées au parcours migratoire (SaglioYatzimirsky \& Wolmark, 2018), nécessiterait des précautions particulières afin de protéger les personnes concernées.

\section{Quels apprentissages pour la formation ?}

Les analyses interactionnelles présentées dans cet article ont permis de mettre en lumière les modalités mises en œuvre pour faire à la diversité linguistique lors des consultations médicales entre locuteurs que ne parlent pas la même langue. Elles ont montré que les répertoires linguistiques des participantes représentent une ressource importante lorsque l'interaction se déroule sans une traduction professionnelle. Dans un contexte où les origines des patients sont de plus en plus variées, il importe de repenser sa pratique professionnelle à partir de la manière de communiquer et d'utiliser les ressources à disposition. Comme nous l'avons vu ici, le travail de reformulation, répétition, adaptation prosodique et simplification linguistique - verbales et incarnées -, bien que complexe et parfois chronophage, se révèle important pour garantir l'accomplissement des objectifs de la visite. Ainsi, faire recours aux propres répertoires linguistiques peut s'avérer utile, même lorsque les compétences ne sont pas solides. À travers l'usage de l'espagnol et de l'italien par exemple, la soignante observée n'a pas seulement pu construire le sens des échanges avec ses interlocuteurs, mais elle a également contribué à gommer, par moments, les asymétries épistémiques concernant les compétences linguistiques manifestées in situ. Cet aspect mériterait d'être développé davantage afin d'en vérifier les retombées de ces pratiques plurilingues sur l'établissement d'un lien de confiance et de proximité entre les locuteurs dont accès aux ressources linguistiques n'est pas égal.

Dans un contexte où les problématiques liées à la situation de précarité et vulnérabilité des patients s'ajoutent à la gestion de la diversité linguistique, l'activité des soignants demeure particulièrement complexe. En effet, ils font face à un ensemble d'activités diversifiées, organisées dans un espace temporel contraint et dans des conditions environnementales parfois compliquées, entre autres par l'absence, nous l'avons vu, d'un aide de traduction adéquate. Ceci est le cas des consultations à la PASS, particulièrement délicates puisque des thématiques conversationnelles non strictement médicales prennent tout de même une place dans l'économie de la visite. Face à ces situations, où des segments de récits concernant des moments du parcours migratoire se produisent, ou d'autres questions non strictement médicales émergent, les médecins se retrouvent à devoir intégrer, dans leur rôle en tant que 'soignant', une fonction d'écoute empathique exigée par la situation. Dans l'extrait 4 analysé antérieurement, où le patient fait mention de son état psychologique fragile, nous avons observé la dimension relationnelle dans l'action du médecin, ayant accompagné de manière 
multimodale (avec sa posture corporelle et la manifestation d'engagement verbal) l'expression du patient, ceci avant d'opérer une réparation, ou de recadrer l'activité. Ces compétences relationnelles, qui prennent forme dans l'ici et maintenant de l'interaction, permettent d'appréhender la complexité de la pratique médicale et d'en saisir les spécificités. Dans ce cadre, il est intéressant de revenir sur la situation décrite dans l'extrait « Why do you leave Nigeria ». Comme nous l'avons vu, le travail interactionnel mené par la soignante afin de connaître la raison du départ du Nigéria par le patient, est le résultat d'une configuration d'éléments (posture, moment dans la consultation, refus du patient, intervention d'un appel téléphonique) non propice au développement de l'activité. Parmi d'autres aspects, nous tenons à évoquer l'importance du contexte séquentiel dans lequel cette activité s'est inscrite. Les études sur les interactions médicales nous disent que chaque phase de la consultation est organisée de manière à favoriser le déroulement de l'activité prévue, les participants s'orientant vers son accomplissement. La question personnelle dont il était question ici a été abordée dans une phase où les participants s'orientent vers les tâches qui lui correspondent (la remise du traitement et les consignes pour l'obtenir) ainsi qu'à la clôture même de la consultation. Pour ces raisons, la manifestation d'intérêt vers le patient/migrant, qui nous semble être le sens ultime de la question, prend ici une coloration différente, presque inopportune.

Plus généralement, l'approche interactionnelle nous a permis d'illustrer la pratique professionnelle dans sa multidimensionnalité, de mettre en lumière les savoir-faire interactionnel et relationnel qui en découlent, dans un cadre où les vulnérabilités des patients appellent à une prise en charge. Les actions de formation que nous avons mises en place (voir Ticca 2018, Ticca et al. 2020) bénéficient de cette approche qui permet de développer auprès de ces acteurs du terrain une conscientisation de la dimension langagière multimodale de la pratique, ceci afin de renforcer leurs compétences cliniques et relationnelles.

\section{Références}

Bischoff, Alexander, Perneger Thomas V., Bovier Patrick A., Loutan Louis, Stalder Hans. (2003). Improving Communication between Physicians and Patients Who Speak a Foreign Language. British Journal of General Practice, 53, p. 541-546.

Cosnier Jacques, Grosjean Michèle, Lacoste Michèle. (éds) (1993). Soins et communication, Lyon, PUL/ARCI.

Drew Paul, Heritage John. (éds) (1998). Talk at Work: Interaction in Institutional Settings, New York: Cambridge University Press.

Duranti Alessandro, Goodwin Charles. (1992). Rethinking Context: Language as an Interactive Phenomenon, Cambridge, Cambridge University Press.

Fillietaz Laurent. (2014). L'interaction langagière : un objet et une méthode d'analyse en formation d'adultes. In Janette Friedrich, Juan Carlos Pita Castro (éds), Recherches en formation des adultes : un dialogue entre concepts et réalité. Dijon: Editions Raisons et Passions, p. 127-162.

Goguikian Ratcliff Betty. (2010). Du bon usage de l'interprète, entre neutralité et implication émotionnelle. In Pascal Singy, Céline Bourquin, Orest Weber (éds), Barrières linguistiques en contexte médical, Lausanne : Cahiers de L'ILSL, p. 39-56.

Heritage John. (2004). Conversation Analysis and Institutional Talk. In Robert Sanders, Kristine L. Fitch (éds), Handbook of Language and Social Interaction, Mahwah NJ, Erlbaum, p. 103-146.

Heritage John, Maynard Douglas (éds) (2006). Communication in medical care, Cambridge, Cambridge University Press. 
Lacoste Michèle. (1980). La vieille dame et le médecin. Etudes de Linguistique Appliquée 37, p. 34-43.

Lacoste Michèle. (1993). Langage et interaction. Le cas de la consultation médicale. In Jacques Cosnier, Michelle Grosjean, Michèle Lacoste (éds), p. 31-61.

Mondada Lorenza. (2006). Interactions en situations professionnelles et institutionnelles : de l'analyse détaillée aux retombées pratiques. Revue française de linguistique appliquée, 2(2), p. 5-16.

Niemants Natacha, Claudio Baraldi, Gavioli Laura. (2015). L'entretien clinique en présence d'un interprète : la traduction comme activité de coordination. Langage et société, 3(3), p. 31-44.

Roberts Celia. (2010). Communicating decisions with a multilingual patient population. Cahiers de l'ILSL, 28, P. 9-25.

Pekarek Doehler Simona, Bangerter Adrian, Geneviève De Weck, Filliettaz Laurent, Ester Gonzalez Martinez, Petitjean Cécile. (éds) (2017). Interactional competences in institutional settings: From school to the workplace, London: Palgrave.

Piccoli Vanessa. (2020). L'hétéro-répétition plurilingue : une pratique pour l'intercompréhension romane ?. Bulletin suisse de linguistique appliquée, 111, p. 4363.

Piccoli Vanessa, Ticca Anna C., Traverso Véronique. (2019). 'Go it's Internet here' : Démarches administratives de personnes précaires ou en demande d'asile. Langage et Société 167(2), p. 81-110.

Saglio-Yatzimirsky Marie-Caroline, Wolmark Laure. (2018). Santé mentale des exilés en France : entre impuissance et créativité, Revue européenne des migrations internationales, 34 (2 et 3), p. 21-27.

Singy Pascal, Bourquin Céline, Weber Orest (éds). (2010). Barrières linguistiques en contexte médical Lausanne : Cahiers de L'ILSL.

Svennevig Jan. (2004). Other-repetition as display of hearing, understanding and emotional stance. Discourse Studies, 6, p. 489-516.

Ticca Anna C. (2018). The interpreter 's activity between complexity and simplification in psychotherapy sessions. inTRAlinea [http://www.intralinea.org/archi ve/article/2312].

Ticca Anna C. (à paraître). Raconter les violences physiques et observer leurs traces dans les consultations médicales pour le droit d'asile. In Nicolas Chambon, Véronique Traverso (éds), Raconter, relater, traduire : paroles de la migration, Lambert Lucas.

Ticca Anna C., Traverso Véronique. (2015a). La bonne information : quand les interprètes corrigent les réponses du patient au docteur dans la consultation médicale. The Interpreters' Newsletter 20, p. 161-174.

Ticca Anna C., Traverso Véronique. (2015b). Traduction orale, interaction et situations sociales. Langage et Société, 153, 3, p. 7-30.

Ticca Anna C., Traverso Véronique. (2017). Parole, voix et corps : lorsque l'interprète et le soignant s'alignent dans les consultations avec migrants. L'autre - clinique, culture et société, 18 (3), p. 304-314.

Ticca Anna C., Lambert Patricia, Traverso Véronique. (2020). Le récit de la migration dans la formation en santé avec les demandeurs d'asile en France, Phronesis 9(3), p. 7792.

Traverso Véronique. (2016). Décrire le français parlée en interaction, Paris : Ophrys. 
Traverso Véronique. (2017). Formulations, reformulations et traductions dans l'interaction : le cas de consultations médicales avec des migrants. Revue française de linguistique appliquée, 22 (2), p. 147-164.

Vinatier Isabelle. (2013). Le travail de l'enseignant : une approche par la didactique professionnelle. Bruxelles : De Broeck.

Weber Orest, Molina Maria Eugenia. (2003). Le point de vue des médiateurs culturels/interprètes. In Patrice Guex, Pascal Singy (éds) Quand la médecine a besoin d'interprètes, Genève : Editions Médecine \& hygiène, p. 85-112.

\section{Conventions de transcription}

La version complète des conventions ICOR est consultable sur le site CORINTE http://icar.univ-lyon2.fr/projets/corinte/.

\begin{tabular}{|c|c|c|c|}
\hline$[1]$ & & $x x x$ & seament inaudible \\
\hline par- & troncation & 八 & intonation montante/ descendantel \\
\hline : & allongement & .h & aspiration \\
\hline (.) & pauses non chronométrées $(<0.2 \mathrm{~s})$ & (il va) & transcription incertaine \\
\hline$(2.2)$ & pauses chronométrées (en sec.) & ${ }^{\circ} \mathrm{bon}^{\circ}$ & voix basse \\
\hline \& & continuation du tour de parole & ALORS & volume augmenté ou autre marque d'insistance \\
\hline $\begin{array}{l}((\text { rire })) \\
\text { (-) }\end{array}$ & $\begin{array}{l}\text { phénomènes non transcrits, commentaires } \\
\text { sourire }\end{array}$ & {$[\ldots]$} & coupure due au transcripteur \\
\hline No & 1 des gestes & & \\
\hline Im. & Dans la colonne des pseudonymes, il indic & e que la & est consacrée à l'insertion d'une image \\
\hline \# & Situe l'emplacement d'une image tirée de I & bande $\mathrm{vi}$ & \\
\hline$(())$ & Description de geste & & \\
\hline
\end{tabular}

